\title{
Torque Control of a Hydrostatic Transmission Using Extended Linearisation Techniques
}

\author{
Robert Prabel and Harald Aschemann \\ Chair of Mechatronics, University of Rostock, Justus-von-Liebig-Weg 6, 18059 Rostock, Germany \\ E-mail: \{robert.prabel, harald.aschemann $\} @$ uni-rostock.de
}

\begin{abstract}
This paper presents a decentralised control approach for the hydraulic motor torque provided by a hydrostatic transmission. Based on a control-oriented model of the hydrostatic transmission, a quasi-linear state-space representation with both state-dependent and input-dependent matrices is derived. Using extended linearisation techniques, a combined feedforward and feedback control is designed. Furthermore, a sliding-mode observer estimates unmeasurable states as well as disturbances. The estimates for the disturbances - an external load torque and a leakage oil volume flow - can be used for a disturbance rejection. The proposed overall control structure is investigated thoroughly in simulations and, afterwards, implemented as well as validated on a dedicated test rig.
\end{abstract}

Keywords: Hydrostatic Transmission, Torque Control, Extended Linearisation, Leakage Estimation, Disturbance Rejection

\section{Introduction and Motivation}

Hydrostatic transmissions (HST) are usually implemented in construction machines, e.g. wheel loaders and excavators, as well as in mining and agricultural applications. Moreover, new application fields have emerged regarding wind turbines, cf. [1,2], and power-split systems, cf. [3], where a precise tracking of angular velocities becomes important regarding a high transmission efficiency. Suitable control concepts for a tracking of the angular velocity of the hydraulic motor are presented in [4] and [5]. Besides these velocity control approaches, it is also possible to control the hydraulic motor torque that is provided by the hydrostatic transmission to the driven vehicle. The design and the validation of such an observer-based nonlinear control structure is presented in this paper.

The set-up of a hydrostatic transmission in construction machines is typically as follows: A prime mover, e.g. an internal

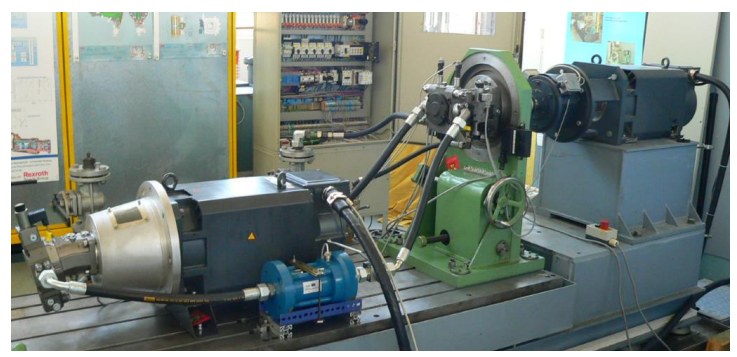

Figure 1: Drive train with a closed-circuit hydrostatic transmission. combustion engine, drives a hydraulic pump with a variable volumetric displacement, which is connected by hydraulic hoses in a closed circuit to the hydraulic motor. Given the overpressure between the high-pressure and the low-pressure side, the hydraulic motor, which also offers a variable volumetric displacement, generates a hydraulic torque. Fig. 1 shows a dedicated test rig for the validation of new control concepts, which is available at the Chair of Mechatronics, University of Rostock. Here, two electric motors are used to represent the prime mover as well as to generate specified disturbances, e.g. driving resistance forces. In Fig. 2, the corresponding structure of the test rig is shown.

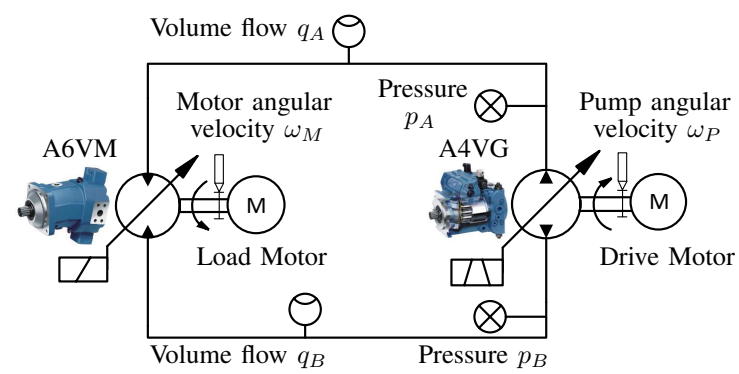

Figure 2: Structure of the dedicated test rig in a closedcircuit configuration consisting of an electric drive motor, a hydraulic pump (A4VG), a hydraulic motor (A6VM), an electric load motor, two hydraulic hoses as well as the instrumentation.

The outline of this paper is as follows: A control-oriented model is derived in Sect. 2. Next, a decentralised control- 
ler based on extended linearisation techniques is derived in Sect. 3. Furthermore, the control structure is extended by a sliding mode observer, see Sect. 4 , to estimate the unmeasured states - tilt angles of the hydraulic pump and motor as well as disturbances, e.g. leakage flows and disturbance torques. Besides, the overall control structure is validated in simulations and experiments, see Sect. 5 and 6. Finally, the paper concludes with a short summary of this contribution and an outlook on further work.

\section{Mechatronic Model of the Hydrostatic Transmission}

A control-oriented model of the hydrostatic transmission provides the necessary information for the control design. Suitable modells for hydraulic applications can be found in [6], [7] and [8]. The mathematical description of the test rig shown in Fig. 1 can be divided in hydraulic and mechanical subsystems. Here, the hydraulic pump is driven by an electric motor that operates in a highly efficient operating point. The corresponding angular velocity of the electric drive motor is chosen as $\omega_{P}=$ const. $>0$.

\subsection{Hydraulic Subsystem}

The hydraulic subsystem includes the hydraulic pump and the hydraulic motor as well as the pressure dynamics in the hydraulic hoses. The corresponding models for the individual hydraulic components are described in the sequel.

\section{Pump Flow Rate}

The pump ideal flow rate $q_{P}$ is determined by a nonlinear function

$$
q_{P}=V_{P}\left(\alpha_{P}\right) n_{P},
$$

with $n_{P}$ as the rotational speed of the pump shaft in rpm. The nonlinear behaviour of the volumetric displacement $V_{P}\left(\alpha_{P}\right)$ is related to the mechanical design based on a tiltable swashplate. A mathematical description according to Fig. 3 leads to

$$
V_{P}\left(\alpha_{P}\right)=N_{P} A_{P} D_{P} \tan \left(\alpha_{P, \max } \cdot \tilde{\alpha}_{P}\right),
$$

with the normalised swashplate angle $\tilde{\alpha}_{P}=\alpha_{P} / \alpha_{P, \max }$. The geometrical parameters are the effective piston area $A_{P}$, the diameter $D_{P}$ of the piston circle and the number $N_{P}$ of pistons inside the pump. The overall pump flow can be described by

$$
q_{P}=\underbrace{\frac{N_{P} A_{P} D_{P}}{2 \pi}}_{\tilde{V}_{P}} \tan \left(\alpha_{P, \max } \cdot \tilde{\alpha}_{P}\right) \omega_{P}=\tilde{V}_{P} \tan \left(\alpha_{P, \max } \cdot \tilde{\alpha}_{P}\right) \omega_{P},
$$

with the pump angular velocity $\omega_{P}$.

\section{Motor Flow Rate}

The used hydraulic motor is of a bent-axis design, see Fig. 4. Therefore, the ideal volume flow rate $q_{M}$ into the hydraulic motor can be described by

$$
q_{M}=V_{M}\left(\alpha_{M}\right) n_{M}
$$

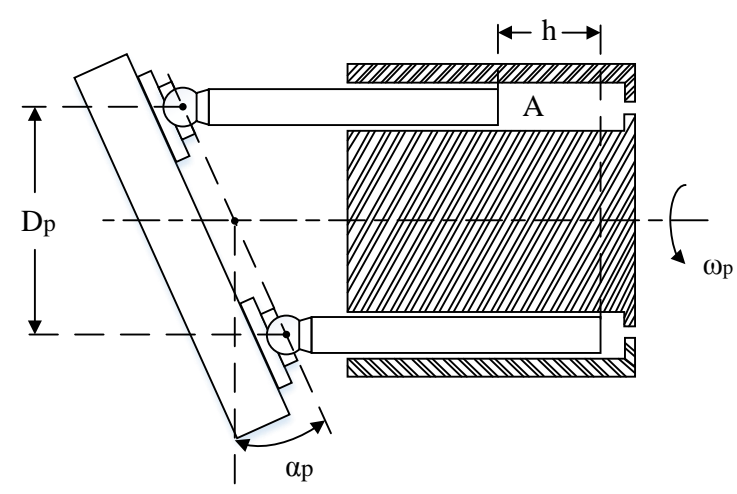

Figure 3: Geometry of the hydraulic pump with a swashplate.

similarly to the pump. In (4), $V_{M}\left(\alpha_{M}\right)$ represents the non-

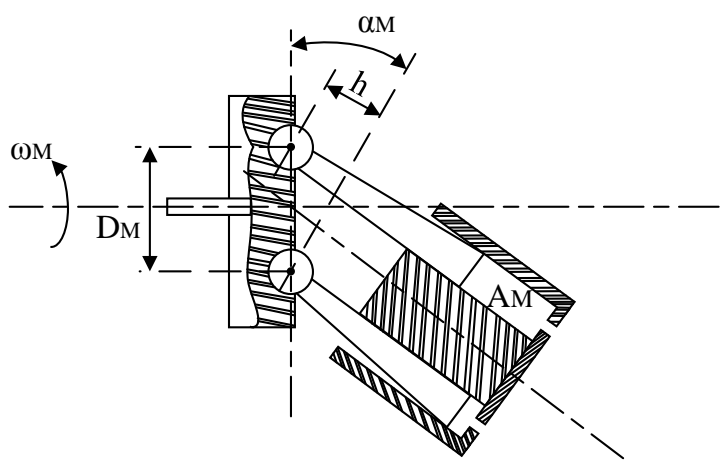

Figure 4: Geometry of the hydraulic motor in bent-axis design.

linear volumetric displacement of the motor and $n_{M}$ the rotational speed of the motor shaft in rpm. With the geometrical parameters $N_{M}, A_{M}$ and $D_{M}$ of the hydraulic motor, the volume flow rate can be stated as

$$
\begin{aligned}
q_{M} & =\underbrace{\frac{N_{M} A_{M} D_{M}}{2 \pi}}_{\tilde{V}_{M}} \sin \left(\alpha_{M, \max } \cdot \tilde{\alpha}_{M}\right) \omega_{M} \\
& =\tilde{V}_{M} \sin \left(\alpha_{M, \max } \cdot \tilde{\alpha}_{M}\right) \omega_{M} .
\end{aligned}
$$

Likewise to the mathematical description of the flow rate of the pump, a normalised bent-axis angle is introduced with $\tilde{\alpha}_{M}=\alpha_{M} / \alpha_{M, \max }$. The constant parameter $\tilde{V}_{M}$ is determined by the mechanical design of the motor.

\section{Pressure Dynamics}

The pressure dynamics of the high-pressure and the lowpressure sides of the hydrostatic transmission are given by

$$
\begin{array}{r}
\dot{p}_{A}=\frac{\beta_{A}}{V_{A}}\left(q_{P}-q_{M}-q_{I}-q_{E, A}\right), \\
\dot{p}_{B}=\frac{\beta_{B}}{V_{B}}\left(-q_{P}+q_{M}+q_{I}-q_{E, B}\right),
\end{array}
$$

with the effective bulk moduli $\beta_{k}, k \in\{A, B\}$ and the total compression volumes $V_{k}, k \in\{A, B\}$, which take into account the hydraulic hoses and the chambers, respectively. 
The volume flow balances for the compression volumes depend on the volume flows $q_{i}, i \in\{P, M\}$ of the pump and the motor, an internal leakage flow $q_{I}$ as well as external leakage flows $q_{E, i}, i \in\{P, M\}$, see Fig. 5. Next, a symmet-

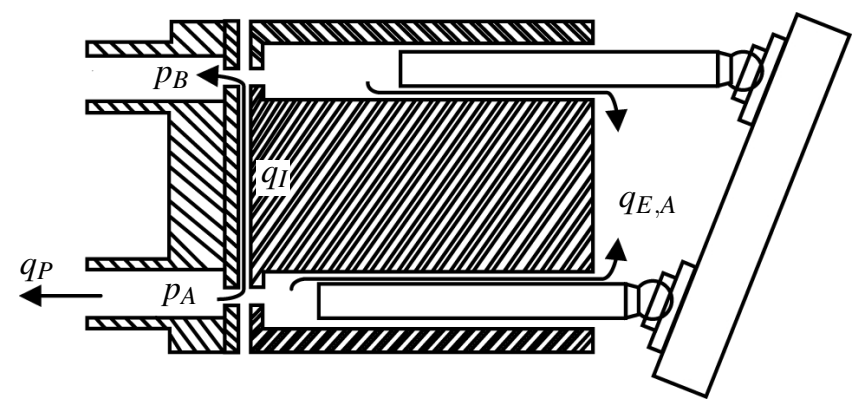

Figure 5: Example for the leakage paths for a axial piston hydraulic pump, with the internal leakage $q_{I}$ and the external leakage $q_{E}$.

ric set-up regarding the high- and low-pressure sides is assumed. The hydraulic capacity with $C_{H}=V / \beta$ can be considered as nearly identical with $C_{A}=C_{B}=: C_{H}$. To reduce the model complexity for the control design, the difference pressure $\Delta p=p_{A}-p_{B}$ is introduced. The corresponding differential equation is given by

$$
\begin{aligned}
\Delta \dot{p}= & \frac{2}{C_{H}}\left(\tilde{V}_{P} \tan \left(\alpha_{P, \max } \cdot \tilde{\alpha}_{P}\right) \omega_{P}-\tilde{V}_{M} \sin \left(\alpha_{M, \max } \cdot \tilde{\alpha}_{M}\right) \omega_{M}\right) \\
& -\frac{q_{u}}{C_{H}}
\end{aligned}
$$

where

$$
q_{u}=2 q_{I}+q_{E, A}-q_{E, B}
$$

denotes a resulting leakage oil flow that acts as a disturbance.

\section{Actuator Dynamics}

It is obvious that an instantaneous change of the displacement of the hydraulic pump as well of the hydraulic motor is impossible. To model such a lag behaviour, first-order lag models are introduced according to

$$
\begin{array}{r}
T_{u P} \dot{\tilde{\alpha}}_{P}+\tilde{\alpha}_{P}=k_{P} u_{P}, \\
T_{u M} \dot{\tilde{\alpha}}_{M}+\tilde{\alpha}_{M}=k_{M} u_{M} .
\end{array}
$$

Here, $T_{u P}$ and $T_{u M}$ represents the corresponding time constants, $k_{P}$ and $k_{M}$ the proportional gains and $u_{P}$ and $u_{M}$ the analogue input voltages of the servo valves. Furthermore, the angles are bounded due to the mechanical design with $\tilde{\alpha}_{P} \in\{-1,1\}$ and $\tilde{\alpha}_{M} \in\left\{\varepsilon_{M}, 1\right\}, \varepsilon_{M}>0$.

\subsection{Mechanical Subsystem}

Typically, hydrostatic transmissions are used in construction machines. In the laboratory environment, see Fig. 1, the hydraulic motor is connected to an electric load motor, which serves for providing specified driving resistances. The set up of the remaining drive train of the test rig is depicted in Fig. 6.

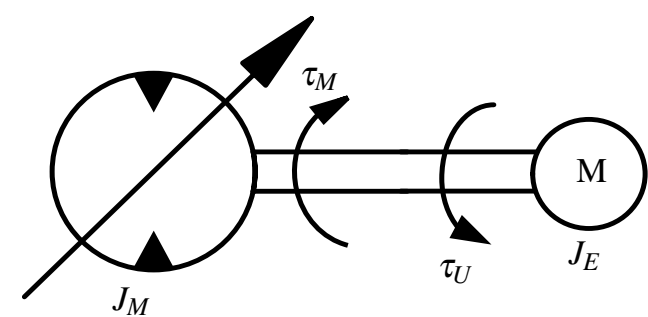

Figure 6: Kinematic structure of the drive train.

A torque balance leads to the equation of motion for the hydraulic pump shaft

$$
J_{V} \dot{\omega}_{M}+d_{v} \omega_{M}=\tau_{M}-\tau_{U}
$$

with $J_{V}=J_{M}+J_{E}$ as the sum of the mass moments of inertia of the hydraulic motor and the electric load motor, which are rigidly connected. Unmodelled disturbances and parameter uncertainties are combined in a lumped disturbance torque $\tau_{U}$. The hydraulic torque of the motor is given by

$$
\tau_{M}=\tilde{V}_{M} \Delta p \sin \left(\alpha_{M, \max } \cdot \tilde{\alpha}_{M}\right)
$$

\subsection{Modelling of the Complete System}

The dynamics of the overall test rig consists of four firstorder differential equations. By introducing the normalised tilt angles $\tilde{\alpha}_{P}$ and $\tilde{\alpha}_{M}$, the difference pressure $\Delta p$ and the angular velocity of the drive shaft $\omega_{M}$ as state variables, the state vector becomes

$$
\boldsymbol{x}=\left[\begin{array}{llll}
\tilde{\alpha}_{P} & \tilde{\alpha}_{M} & \Delta p & \omega_{M}
\end{array}\right]^{T}
$$

and the corresponding nonlinear state-space representation results in

$$
\left[\begin{array}{c}
\dot{\tilde{\alpha}}_{P} \\
\dot{\tilde{\alpha}}_{M} \\
\Delta \dot{p} \\
\dot{\omega}_{M}
\end{array}\right]=\left[\begin{array}{c}
-\frac{1}{T_{u P}} \tilde{\alpha}_{P}+\frac{k_{P}}{T_{u P}} u_{P} \\
-\frac{1}{T_{u M}} \tilde{\alpha}_{M}+\frac{k_{M}}{T_{u M}} u_{M} \\
{\left[\frac{2}{C_{H}} \tilde{V}_{P} \tan \left(\alpha_{P, \max } \cdot \tilde{\alpha}_{P}\right) \omega_{P}\right.} \\
\left.-\frac{2}{C_{H}} \tilde{V}_{M} \sin \left(\alpha_{M, \max } \cdot \tilde{\alpha}_{M}\right) \omega_{M}-\frac{q_{u}}{C_{H}}\right] \\
-\frac{d_{V}}{J_{V}} \omega_{M}+\frac{\tilde{V}_{M}}{J_{V}} \sin \left(\alpha_{M, \max } \cdot \tilde{\alpha}_{M}\right)-\frac{\tau_{U}}{J_{V}}
\end{array}\right] .
$$

The input voltages of the proportional valves for the actuation of the hydraulic pump and motor are used as control inputs

$$
\boldsymbol{u}=\left[\begin{array}{ll}
u_{P} & u_{M}
\end{array}\right]^{T}
$$

\section{Nonlinear Control Design}

The proposed control structure is based on a decentralised approach. In a first control loop, the normalised tilt angle of the hydraulic motor $\tilde{\alpha}_{M}$ is controlled by a flatness-based approach. The second control loop is responsible for the torque control of the hydraulic motor by using extended linearisation techniques. 


\subsection{Flatness-Based Control of the Tilt Angle of the Hy- draulic Motor}

The control design for $\tilde{\alpha}_{M}$ is performed using a flatness-based approach, see [9]. Thereby, the inverse dynamics results in

$$
u_{M}=\frac{\tilde{\alpha}_{M}+v_{M} T_{u M}}{k_{M}},
$$

with the stabilising control law for the error dynamics

$$
v_{M}=\dot{\tilde{\alpha}}_{M d}+k_{\alpha 0} e_{\tilde{\alpha}_{M}}+k_{\alpha I} \cdot \int_{0}^{t} e_{\tilde{\alpha}_{M}} d \tau
$$

Here, $e_{\tilde{\alpha}_{M}}=\tilde{\alpha}_{M, d}-\tilde{\alpha}_{M}$ represents the tracking error of the normalised tilt angle. The error dynamics $e_{\tilde{\alpha}_{M}}$ is parametrised with positive coefficients $k_{\alpha 0}>0$ and $k_{\alpha I}>0$.

\subsection{State Feedback Design Using Extended Linearisa- tion Techniques}

Linear control approaches like eigenvalue placement and LQR design are well known and often used for linear statespace systems.In this paper, the eigenvalue placement method is extended to the nonlinear case, which is corresponds to extended linearisation design techniques, cf. [10]. Therefore, the system is written in the form of a quasi-linear state-space representation

$$
\begin{aligned}
\dot{\boldsymbol{x}}(t) & =\boldsymbol{A}\left(\omega_{p}, \tilde{\alpha}_{P}\right) \boldsymbol{x}(t)+\boldsymbol{b} u(t)+\boldsymbol{e} z(t) \\
y(t) & =\boldsymbol{c}^{T}\left(\tilde{\alpha}_{M}\right) \boldsymbol{x}(t)
\end{aligned}
$$

Here, the system matrix $\boldsymbol{A}=\boldsymbol{A}\left(\omega_{p}, \tilde{\alpha}_{P}\right)$ depends on the angular velocity as well on the tilt angle of the hydraulic pump. Furthermore, the nonlinear output vector $c^{T}=c^{T}\left(\tilde{\alpha}_{M}\right)$ is affected by the tilt angle of the hydraulic motor. Note that the corresponding state equation can be neglected, and $\omega_{M}$ is considered as a gain-scheduling parameter. The parametrisation of the quasi-linear form results in

$$
\begin{aligned}
\dot{\boldsymbol{x}} & =\underbrace{\left[\begin{array}{cc}
\frac{-1}{T_{u P}} & 0 \\
\frac{2 \tilde{V}_{P} \omega_{P}}{C_{H}} \frac{\operatorname{si}\left(\alpha_{P, \max } \cdot \tilde{\alpha}_{P}\right) \cdot \alpha_{P, \max }}{\cos \left(\alpha_{P, \max } \cdot \tilde{\alpha}_{P}\right)} & 0
\end{array}\right]}_{\boldsymbol{A}} \underbrace{\left[\begin{array}{c}
\tilde{\alpha}_{P} \\
\Delta p
\end{array}\right]}_{\boldsymbol{x}} \\
+ & \underbrace{\left[\begin{array}{c}
\frac{k_{P}}{T_{u P}} \\
0
\end{array}\right]}_{\boldsymbol{b}} u_{P}+\underbrace{\left[\begin{array}{c}
0 \\
\frac{1}{C_{H}}
\end{array}\right]}_{\boldsymbol{e}} \underbrace{-2 \tilde{V}_{M} \omega_{M} \sin \left(\alpha_{M, \max } \cdot \tilde{\alpha}_{M}\right)-q_{U}}_{z} .
\end{aligned}
$$

The si-function is determined by $\operatorname{si}\left(\tilde{\alpha}_{P}\right)=\sin \left(\tilde{\alpha}_{P}\right) / \tilde{\alpha}_{P}$, with $\operatorname{si}\left(\tilde{\alpha}_{P}=0\right)=1$. The nonlinear output equation results in

$$
y=\tau_{M}=\underbrace{\left[\begin{array}{lll}
0 & \tilde{V}_{M} \sin \left(\alpha_{M, \max } \cdot \tilde{\alpha}_{M}\right)
\end{array}\right]}_{\boldsymbol{c}^{T}\left(\tilde{\alpha}_{M}\right)} \boldsymbol{x} .
$$

A subsequent analysis regarding the controllability leads to the following condition: The angular velocity $\omega_{P}$ must not vanish; this holds because the drive motor operates at a constant angular velocity $\omega_{P} \neq 0$. The tilt angle of the motor is confined to strictly positive values $\tilde{\alpha}_{M} \in\left\{\varepsilon_{M}, 1\right\}, \varepsilon_{M}>0$.

Based on the quasi-linear representation (18), an eigenvalue placement is performed to calculate the state-dependent feedback gains $\boldsymbol{k}^{T}(\boldsymbol{x})$. In a next step, a feedforward control $u_{F F}$ is designed to achieve steady state accuracy. For a further improvement of the tracking behaviour, a dynamic disturbance rejection is introduced. The overall control input can be calculated as the sum of all three control actions

$$
u_{P}=-\boldsymbol{k}^{T}(\boldsymbol{x}) \boldsymbol{x}+u_{F F}-u_{D C}
$$

\section{Eigenvalue Assignment Using Extended Linearisation}

The quasi-linear dynamical system to be stabilised is given by (18) - characterised by a state-dependent system matrix $\boldsymbol{A}=\boldsymbol{A}\left(\omega_{p}, \tilde{\alpha}_{P}\right)$ and an input vector $\boldsymbol{b}$. The feedback control design in the form of an eigenvalue placement involves the symbolic computation of a state-dependent gain vector $\boldsymbol{k}^{T}=\boldsymbol{k}\left(\omega_{p}, \tilde{\alpha}_{P}\right)^{T}$ by a comparison of the desired characteristic polynomial of the closed-loop system

$$
p_{c d}(s)=\left(s-s_{c 1}\right)\left(s-s_{c 2}\right),
$$

specifying two desired eigenvalues $s_{c i}, i=\{1,2\}$, with the following characteristic equation

$$
p_{c}(s)=\operatorname{det}\left(s \boldsymbol{I}_{2}-\boldsymbol{A}_{c}\left(\boldsymbol{k}^{T}\right)\right) .
$$

In (22), $\boldsymbol{I}_{2}$ is the $2 \times 2$ identity matrix and $\boldsymbol{A}_{c}$ the closed-loop system matrix

$$
\boldsymbol{A}_{c}:=\boldsymbol{A}-\boldsymbol{b} \boldsymbol{k}^{T}\left(\omega_{p}, \tilde{\alpha}_{P}\right)
$$

The state feedback is calculated by $u_{F B}=-\boldsymbol{k}^{T}\left(\omega_{p}, \tilde{\alpha}_{P}\right) \boldsymbol{x}$ with the state- and parameter-dependent gain vector

$$
\boldsymbol{k}\left(\omega_{p}, \tilde{\alpha}_{P}\right)=\left[\begin{array}{c}
\frac{-\left(\left(s_{c 1}+s_{c 2}\right) T_{u P}+1\right)}{k_{P}} \\
\frac{s_{c 1} s_{c 2} C_{H} \cos \left(\alpha_{P, \max } \cdot \tilde{\alpha}_{P}\right) T_{u P}}{2 \tilde{V}_{P} \omega_{P} \operatorname{si}\left(\alpha_{P, \max } \cdot \tilde{\alpha}_{P}\right) \alpha_{P, \max } k_{P}}
\end{array}\right] .
$$

\section{Feedforward Control Design Using Extended Linearisa- tion}

For the feedforward control design, the hydraulic torque $\tau_{M}$ generated by the hydraulic motor according to (11) is considered as the controlled variable. Thus, the nonlinear output equation is given by (19) and depends on $\tilde{\alpha}_{M}$. The command transfer function can be calculated as

$$
G_{b}(s)=\frac{Y(s)}{U_{F F}(s)}=c^{T}\left(s \boldsymbol{I}-\boldsymbol{A}_{c}\right)^{-1} \boldsymbol{b}=\frac{b_{0}}{N(s)} .
$$

Obviously, the numerator of the control transfer function contains no transfer zero. The main idea of the feedforward control design is the modification of the numerator of the control transfer function by introducing a polynomial ansatz for the feedforward control action in the Laplace domain according to

$$
U_{F F}(s)=\left[k_{V 0}+k_{V 1} \cdot s+k_{V 2} \cdot s^{2}\right] Y_{d}(s) .
$$


For its implementation, the desired trajectory $y_{d}(t)=\tau_{M, d}(t)$ as well as the first two time derivatives are available from a state variable filter. The feedforward gains can be computed from a comparison of the corresponding coefficients in the numerator as well as the denominator polynomials of

$$
\frac{Y(s)}{Y_{d}(s)}=\frac{b_{0} \cdot\left[k_{V 0}+k_{V 1} \cdot s+k_{V 2} \cdot s^{2}\right]}{a_{0}+a_{1} \cdot s+a_{2} \cdot s^{2}}
$$

according to

$$
a_{i}=b_{0} \cdot k_{V i}, \text { with } i=0,1, n=2 .
$$

The feedforward control is evaluated in the time domain with

$$
u_{F F}=k_{V 0} \tau_{M, d}+k_{V 1} \dot{\tau}_{M, d}+k_{V 2} \ddot{\tau}_{M, d}
$$

\section{Dynamic Disturbance Compensation Using Extended Linearisation}

The disturbance $z$ in (18) depends on the unknown leakage flow $q_{U}$, which has to be estimated, and the states $\omega_{M}$ and $\tilde{\alpha}_{M}$. This disturbance has to be compensated to achieve an acceptably small tracking error. The disturbance transfer function from the disturbance input to the controlled output becomes

$$
G_{e}(s)=\frac{Y(s)}{Z(s)}=c^{T}\left(s \boldsymbol{I}-\boldsymbol{A}_{c}\right)^{-1} \boldsymbol{e} .
$$

For an ideal disturbance compensation, the condition

$$
Y(s)=G_{b}(s) \cdot U_{c}(s)+G_{e}(s) \cdot Z(s) \stackrel{!}{=} 0
$$

has to be fulfilled exactly. As an approximation, an ansatz function $G_{c}(s)$ for the dynamic disturbance compensation is used according to

$$
U_{D C}(s)=G_{d} c(s) \cdot Z(s)=\left[k_{d c 0}+k_{d c 1} \cdot s\right] \cdot Z(s) .
$$

This ansatz function requires values for the disturbance and its first time derivative. By inserting (32) in (31), the design condition becomes

$$
0 \stackrel{!}{=} \underbrace{Z(s)}_{\neq 0}[\underbrace{G_{b}(s) \cdot G_{c}(s)+G_{e}(s)}_{!}] .
$$

For an approximate dynamic disturbance compensation, the corresponding ansatz coefficients are chosen in such a way that the first two coefficients in the nominator polynomial vanish. For the evaluation of the dynamic disturbance compensation

$$
u_{D C}=k_{d c 0} z+k_{d c 1} \dot{z}
$$

the required time derivative of the lumped disturbance $\dot{z}$ is calculated by real differentiation.

\section{Sliding Mode State and Disturbance Ob- server}

Regarding the state variables of the test rig, see (12), only the difference pressure and the angular velocity of the hydraulic motor are measured $\boldsymbol{y}_{m}=\left[\begin{array}{ll}\Delta p & \omega_{M}\end{array}\right]^{T}$. However, it can be seen in that the remain unmeasured states and disturbances $\boldsymbol{x}_{1}=\left[\begin{array}{llll}\tilde{\alpha}_{P} & \tilde{\alpha}_{M} & q_{U} & \tau_{U}\end{array}\right]^{T}$ are needed for calculation of the control inputs e.g. $(20,34)$. The sliding mode observer design, cf. [11] for the case of a linear system, is based on the extended system model with integrators as disturbance models

$$
\dot{z}_{S}=\left[\begin{array}{c}
\dot{\tau}_{U} \\
\dot{q}_{U}
\end{array}\right]=\left[\begin{array}{l}
0 \\
0
\end{array}\right] .
$$

Following the idea of extended linearisation, which is already used in Sect. 3, the extended system with the corresponding state vector

$$
\boldsymbol{x}_{e}=\left[\begin{array}{ll:l}
\boldsymbol{x}_{1}{ }^{T} & \boldsymbol{y}_{m}{ }^{T}
\end{array}\right]^{T}=\left[\begin{array}{llll:ll}
\tilde{\alpha}_{P} & \tilde{\alpha}_{M} & \tau_{U} & q_{U} & \Delta p & \omega_{M}
\end{array}\right]^{T}
$$

is rewritten in a quasi-linear form with a state-dependent system matrix $\boldsymbol{A}_{e}\left(\tilde{\alpha}_{P}, \tilde{\alpha}_{M}, \omega_{M}\right)$

$$
\begin{aligned}
\dot{\boldsymbol{x}}_{e} & =\boldsymbol{A}_{e}\left(\tilde{\alpha}_{P}, \tilde{\alpha}_{M}, \omega_{M}\right) \boldsymbol{x}_{e}+\boldsymbol{B}_{e} \boldsymbol{u} \\
\boldsymbol{y}_{m} & =\boldsymbol{C}_{e} \boldsymbol{x}_{e} .
\end{aligned}
$$

The extended input and output matrices are denoted as $\boldsymbol{B}_{e}$ and $\boldsymbol{C}_{e}$, respectively. The observability can be easily confirmed by point-wise checking the observability matrix of the quasilinear system, which depends on both $\tilde{\alpha}_{M}$ and $\omega_{M}$. For the observer design, the state-space representation is written as

$$
\left[\begin{array}{c}
\dot{\boldsymbol{x}}_{1} \\
\dot{\boldsymbol{y}}_{m}
\end{array}\right]=\left[\begin{array}{ll}
\boldsymbol{A}_{11} & \boldsymbol{A}_{12} \\
\boldsymbol{A}_{21} & \boldsymbol{A}_{22}
\end{array}\right]\left[\begin{array}{l}
\boldsymbol{x}_{1} \\
\boldsymbol{y}_{m}
\end{array}\right]+\left[\begin{array}{l}
\boldsymbol{B}_{1} \\
\boldsymbol{B}_{2}
\end{array}\right] \boldsymbol{u} .
$$

The observer has the form

$$
\begin{aligned}
{\left[\begin{array}{c}
\dot{\hat{\boldsymbol{x}}}_{1} \\
\hat{\boldsymbol{y}}_{m}
\end{array}\right]=} & {\left[\begin{array}{ll}
\boldsymbol{A}_{11} & \boldsymbol{A}_{12} \\
\boldsymbol{A}_{21} & \boldsymbol{A}_{22}
\end{array}\right]\left[\begin{array}{c}
\hat{\boldsymbol{x}}_{1} \\
\hat{\boldsymbol{y}}_{m}
\end{array}\right]+\left[\begin{array}{c}
\boldsymbol{B}_{1} \\
\boldsymbol{B}_{2}
\end{array}\right] } \\
& -\left[\begin{array}{c}
\boldsymbol{G}_{1} \\
\boldsymbol{G}_{2}
\end{array}\right]\left(\hat{\boldsymbol{y}}_{m}-\boldsymbol{y}_{m}\right)+\left[\begin{array}{c}
\boldsymbol{L} \\
-\boldsymbol{I}
\end{array}\right] \boldsymbol{v},
\end{aligned}
$$

where $(\hat{\cdot})$ represents the estimated values. $\boldsymbol{G}_{1}$ and $\boldsymbol{G}_{2}$ denotes Luenberger-type gain matrices, $\boldsymbol{L}$ gain matrix for the discontinuous switching part, which is defined by the vector $\boldsymbol{v}$ according to

$$
\boldsymbol{v}=\left[\begin{array}{c}
M_{1} \operatorname{sign}(\Delta \hat{p}-\Delta p) \\
M_{2} \operatorname{sign}\left(\hat{\omega}_{M}-\omega_{M}\right)
\end{array}\right]
$$

Here, $M_{1,2}$ represent positive, constant gains. Considering the definition $\boldsymbol{e}_{1}=\hat{\boldsymbol{x}}_{1}-\boldsymbol{x}_{1}$ and $\boldsymbol{e}_{y_{m}}=\hat{\boldsymbol{y}}_{m}-\boldsymbol{y}_{m}$ and introducing a new error variable $\overline{\boldsymbol{e}}_{1}=\boldsymbol{e}_{1}+\boldsymbol{L} \boldsymbol{e}_{y_{m}}$, the resulting estimation error dynamics becomes

$$
\left[\begin{array}{c}
\dot{\boldsymbol{e}}_{1} \\
\dot{\boldsymbol{e}}_{y_{m}}
\end{array}\right]=\left[\begin{array}{ll}
\overline{\boldsymbol{A}}_{11} & \overline{\boldsymbol{A}}_{12} \\
\boldsymbol{A}_{21} & \overline{\boldsymbol{A}}_{22}
\end{array}\right]\left[\begin{array}{c}
\overline{\boldsymbol{e}}_{1} \\
\boldsymbol{e}_{y_{m}}
\end{array}\right]+\left[\begin{array}{c}
\mathbf{0} \\
-\boldsymbol{I}
\end{array}\right] \boldsymbol{v},
$$

where the submatrices are given by

$$
\begin{aligned}
& \overline{\boldsymbol{A}}_{11}=\boldsymbol{A}_{11}+\boldsymbol{L} \boldsymbol{A}_{21}, \\
& \overline{\boldsymbol{A}}_{12}=\boldsymbol{A}_{12}-\overline{\boldsymbol{A}}_{11} \boldsymbol{L}-\boldsymbol{G}_{1}+\boldsymbol{L}\left(\boldsymbol{A}_{22}-\boldsymbol{G}_{2}\right), \\
& \overline{\boldsymbol{A}}_{22}=\boldsymbol{A}_{22}-\boldsymbol{G}_{2}-\boldsymbol{A}_{21} \boldsymbol{L} .
\end{aligned}
$$




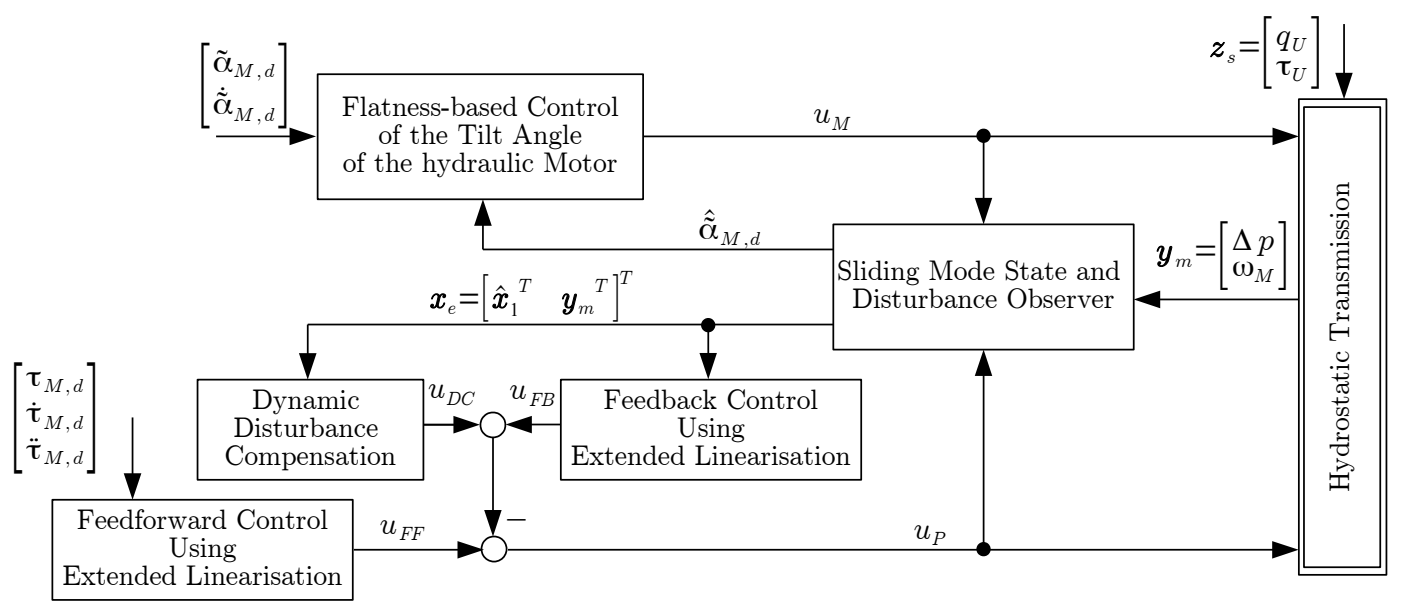

Figure 7: Block diagram of the implemented control structure.

$\overline{\boldsymbol{A}}_{12}=\mathbf{0}$ can be achieved by proper choice of the gain matrix $\boldsymbol{G}_{1}$. In the case of $\boldsymbol{v}=\mathbf{0}$, asymptotic stability of the error dynamics (42) is obtained by choosing the gain matrices $\boldsymbol{L}$ and $\boldsymbol{G}_{2}$ according to

$$
\begin{aligned}
\boldsymbol{A}_{11}+\boldsymbol{L} \boldsymbol{A}_{21} & =\overline{\boldsymbol{A}}_{11}^{*}, \\
\boldsymbol{A}_{22}-\boldsymbol{G}_{2}-\boldsymbol{A}_{21} \boldsymbol{L} & =\overline{\boldsymbol{A}}_{22}^{*},
\end{aligned}
$$

where $\overline{\boldsymbol{A}}_{11}^{*}$ and $\overline{\boldsymbol{A}}_{22}^{*}$ denote asymptotically stable matrices with the following characteristic polynomials

$$
\begin{aligned}
& \operatorname{det}\left(s \boldsymbol{I}-\overline{\boldsymbol{A}}_{11}^{*}\right) \stackrel{!}{=}\left(s-s_{B 1}\right)\left(s-s_{B 2}\right)\left(s-s_{B 3}\right)\left(s-s_{B 4}\right), \\
& \operatorname{det}\left(s \boldsymbol{I}-\overline{\boldsymbol{A}}_{22}^{*}\right) \stackrel{!}{=}\left(s-s_{B 5}\right)\left(s-s_{B 6}\right) .
\end{aligned}
$$

The additional switching input $\boldsymbol{v}$ provides robustness against certain classes of model uncertainty. Considering the chattering caused by the discontinuous components, the tanh function is used in the implementation instead of the sign function.

\section{Simulation Results}

In this section, a simulation study of the proposed robust nonlinear control in combination with the sliding mode state and disturbance observer for the hydrostatic transmission is demonstrated. The implemented control structure is depicted in Fig. 7. To be more realistic, the parametrisation of the leakage flow is assumed to be proportional to the pressure difference according to

$$
q_{U}=1 \cdot 10^{-12} \Delta p
$$

whereas the disturbance torque is given by

$$
\tau_{U}=0.1 J_{V} \dot{\omega}_{M}+7 \tanh \left(\frac{\omega_{M}}{0.1}\right) .
$$

In addition to the disturbance models, a measurement noise is added to the difference pressure $\Delta p$ and the motor angular velocity $\omega_{M}$, which are the only measurable state variables at the test rig.

The obtained results from the simulation are depicted in the following figures. At first, the tracking performance of $\tilde{\alpha}_{M}$ and $\tau_{\text {hyd }}$ is investigated. Fig. 8 shows the desired and the simulated trajectory of the hydraulic motor torque, which match well. In addition, the tracking performance for the control

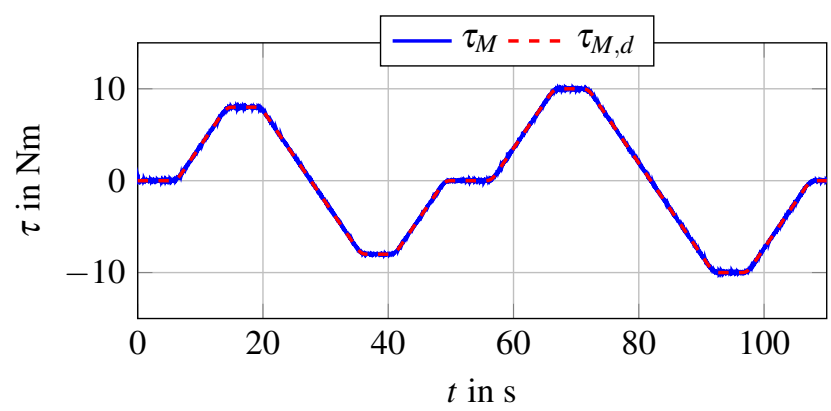

Figure 8: Tracking behaviour of the hydraulic motor torque (simulation results).

loop for the normalised tilt angle of the hydraulic motor is presented in Fig. 9, which indicates an highly accurate tracking behaviour. As a result of the balance of momentum at the

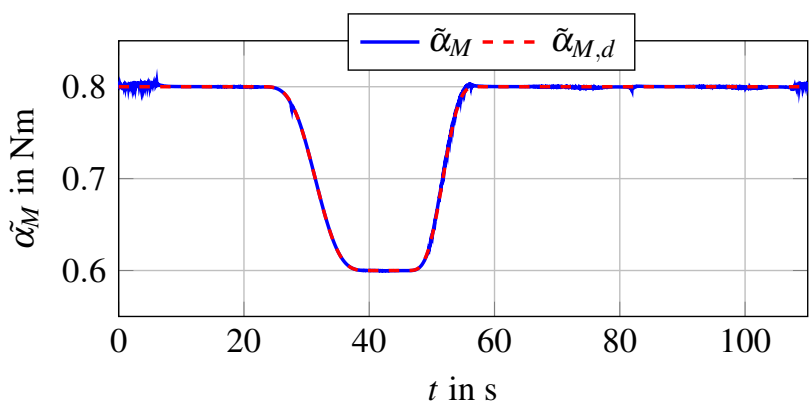

Figure 9: Comparison of desired and simulated values of the normalised tilt angle of the hydraulic motor (simulation results).

hydraulic motor, the related angular velocity $\omega_{M}$ is obtained as shown Fig. 10.

The next figures point out the benefits of the disturbance es- 


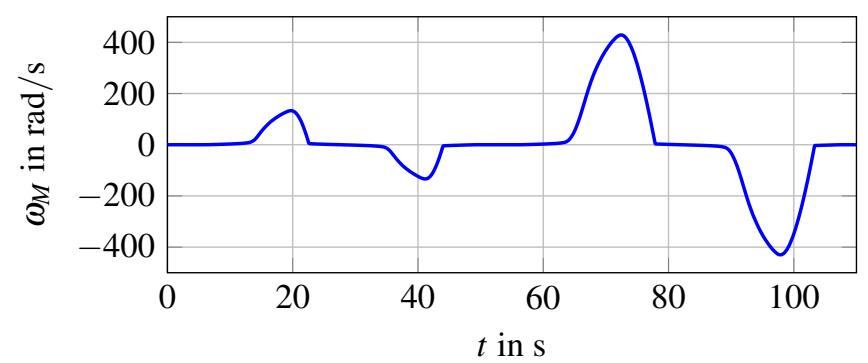

Figure 10: Angular velocity of the hydraulic motor (simulation results).

timation by the sliding mode observer. The modelled disturbance torque as well as the estimated torque are shown in Fig. 11. It becomes obvious that the observer is capable of reconstructing this unknown disturbance. The same holds for the other disturbance, the leakage flow, which is depicted in Fig. 12. Given the positive results from the simulations, the control approach is implemented and validated at the test rig, see Fig. 1.

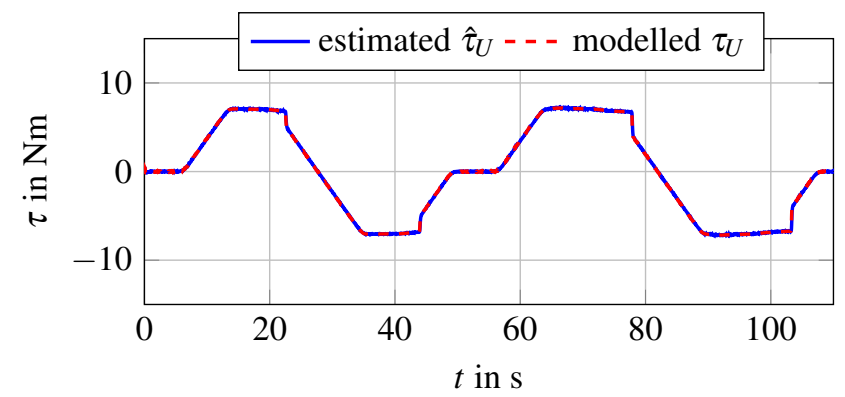

Figure 11: Comparison of the simulated and estimated disturbance torques (simulation results).

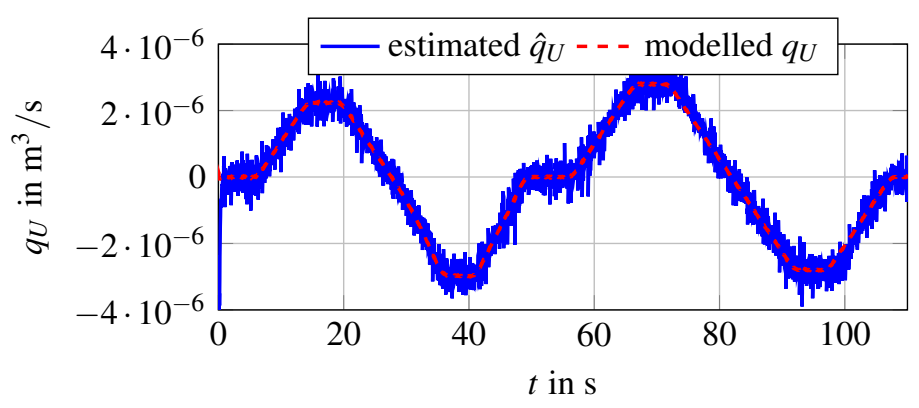

Figure 12: Comparison of the simulated and estimated leakage flow rates (simulation results).

\section{Experimental Results}

In the sequel, the experimental results are presented using the implementation of the control structure according to Fig. 7. A LabVIEW real-time environment is used to operate the test rig. The electric motor that drives the hydraulic pump has at a constant angular velocity of $\omega_{P}=700 \mathrm{rpm}=$ const. This is achieved by an underlying velocity control on a current con- verter. However, for the operation of the electric load motor two alternative control scenarios - either torque mode or velocity mode - are considered to validate the proposed control structure. Note that the torque of the hydraulic motor $\tau_{M}$ is not measured. This value is calculated by (11), where the estimated value of motor tilt angle $\hat{\tilde{\alpha}}_{M}$ is used.

\section{Load Motor Operates in a Torque Mode}

In the torque mode, an underlying torque control on the current converter is employed for the electric load motor. Here, the desired load torque is chosen as $\tau_{U}=0 \mathrm{Nm}$. In Fig. 13, the tracking behaviour of the normalised tilt angle of the hydraulic motor $\tilde{\alpha}_{M}$, which is controlled in the first loop, is depicted. As the motor tilt angle $\tilde{\alpha}_{M}$ is not measurable, the corresponding estimated value is plotted.

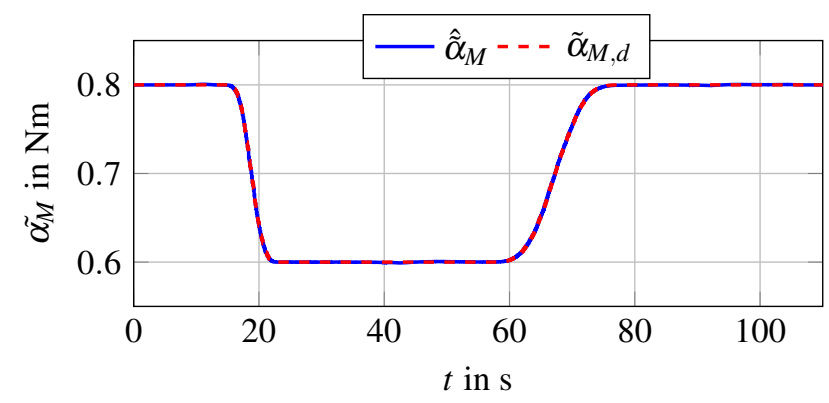

Figure 13: Comparison of actual and desired values of the normalised tilt angle of the hydraulic motor (experimental results).

Fig. 14 shows the tracking behaviour of the hydraulic torque provided by the hydraulic motor, which is controlled in the second loop. Due to the torque balance at the hydraulic motor, the angular velocity of the drive side $\omega_{M}$ varies, see Fig. 15.

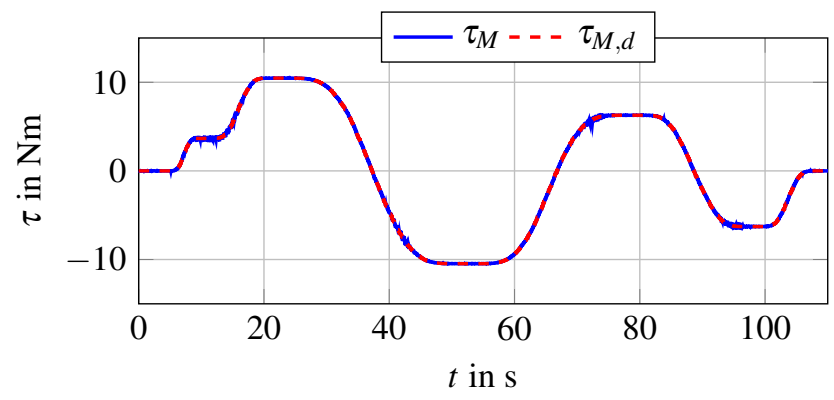

Figure 14: Comparison of actual and desired values of the torque of the hydraulic motor (experimental results, electric load motor operates in a torque mode).

\section{Load Motor Operates in a Velocity Mode}

In the second scenario the electric load motor operates at a constant velocity $\omega_{M}=200 \mathrm{rpm}=$ const. The necessary control for the electric load motor is provided by a corresponding current converter. The obtained tracking behaviour of the hydraulic motor torque, which is characterized by only small er- 


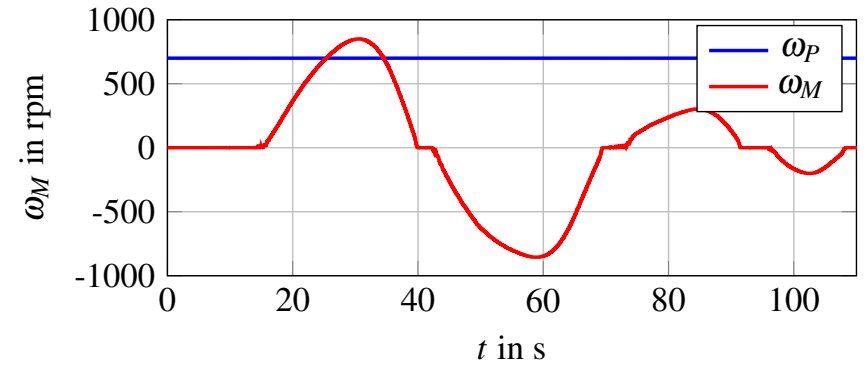

Figure 15: Angular velocities of the electric motors: constant angular velocity at the drive motor and varying angular velocity at the load motor (experimental results, electric load motor operates in a torque mode).

rors, is presented in Fig. 16. Furthermore, the corresponding pressures are depicted in Fig. 17.

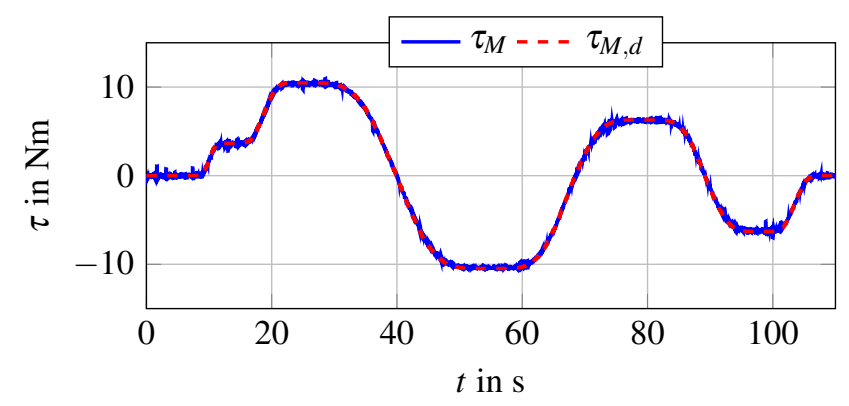

Figure 16: Comparison of actual and desired values of the hydraulic motor torque in the velocity mode (experimental results, electric load motor operates in a velocity mode).

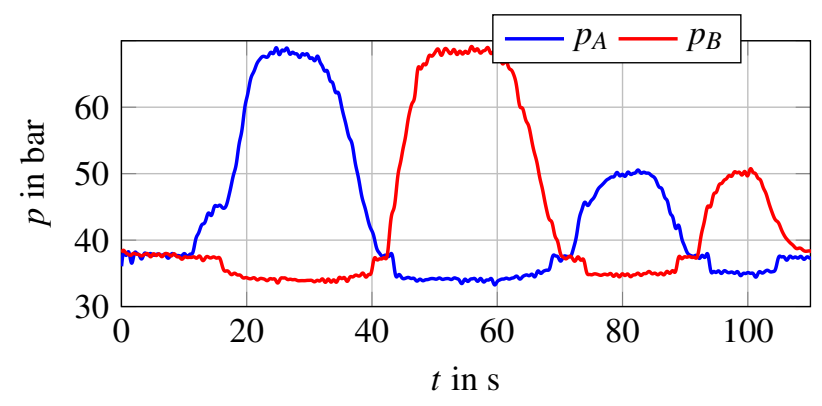

Figure 17: Pressures in both hydraulic hoses during tracking of the torque of the hydraulic motor (experimental results, electric load motor operates in a velocity mode).

\section{Conclusions and Outlook on Further Work}

Based on a decentralised control structure of the hydrostatic transmission, an innovative decentralised nonlinear control structure has been designed. The the hydraulic motor torque of the hydrostatic transmission is controlled using extend linearisation techniques, whereas the control of normalised tilt angle of the hydraulic motor is realized by a flatness-based approach. Moreover, a sliding mode observer - designed by using extended linearisation techniques as well - is introduced to robustly estimate unmeasurable system states and unknown disturbances. The proposed cascaded control structure has been successfully validated in simulations as well by the experimental results.

To further increase the accuracy of the identified mechatronic model of the test-rig, a torque sensor will be installed between the hydraulic motor and the electric load motor. With this future enhancement of the test rig, the tracking behaviour of the controlled hydrostatic transmission is expected to be improved.

\section{References}

[1] B. Dolan and H. Aschemann. Control of a Wind Turbine With a Hydrostatic Transmission - An Extended Linearisation Approach. In 17th Int. Conf. on Methods and Models in Automation and Robotics (MMAR), pages 445-450, Poland, 2012.

[2] N.F.B. Diepeveen and A.J. Laguna. Dynamics Modelling of Fluid Power Transmissions for Wind Turbine. In EWEA Offshore, Netherland, 2011.

[3] H. Schulte and P. Gerland. Control-Oriented Modeling of Hydrostatic Power-Split CVTs Using Takagi-Sugeno Fuzzy Models. In 7th Conf. of the European Society for Fuzzy Logic and Technology (EUSFLAT), pages 797804, France, 2011.

[4] H. Sun and H. Aschemann. Quasi-Continuous Sliding Mode Control Applied to a Hydrostatic Transmission. In European Control Conference (ECC), Austria, 2015.

[5] H. Sun, R. Prabel, and H. Aschemann. Cascaded Control Design for the Tracking Control of a Hydrostatic Transmission Based on a Sliding mode State and Disturbance Observer. In 21st Int. Conf. on Methods and Models in Automation and Robotics (MMAR), pages 432-437, Poland, Aug 2016.

[6] M. Jelali and A. Kroll. Hydraulic Servo-Systems: Modelling, Identification and Control. Springer-Verlag, UK, 2003.

[7] P. Rohner. Industrial Hydraulic Control: A Textbook for Fluid Power Technicians. John Wiley \& Sons, 2004.

[8] A. Kugi, K. Schlacher, H. Aitzetmueller, and G. Hirmann. Modelling and Simulation of a Hydrostatic Transmission with Variable-Displacement Pump. In Mathematics and Computers in Simulation, volume 53, pages 409-414, 2000.

[9] M. Fliess, J. Levine, P. Martin and P. Rouchon. Flatness and Defect of Nonlinear Systems: Introductory Theory and Examples. In Int. J. Control, volume 61, pages 1327-1361, 1995.

[10] B. Friedland. Advanced Control System Design. Prentice Hall, 1996.

[11] C. Edwards and S.K. Spurgeon. Sliding Mode Control: Theory and Applications. Taylor \& Francis Ltd, 1998. 